\title{
Ferroportin and hepcidin: a new hope in diagnosis, prognosis, and therapy for breast cancer
}

lgor P Pogribny

\begin{abstract}
Breast cancer is the most prevalent malignancy in women. The success of breast cancer treatment relies on the ability to detect the disease and correct molecular abnormalities at an early stage of disease development. A recent article describes a marked decrease in the levels of ferroportin in breast cancer. More importantly, the presented results demonstrate convincingly the incredible diagnostic and prognostic value of ferroportin and hepcidin gene expression in breast cancer and suggest that determination of these two molecular markers may be used as guidance toward individualized therapy for breast cancer patients.
\end{abstract}

\section{Background}

Iron is essential for major fundamental cellular processes in all living organisms. During recent years, interest in the field of iron metabolism has been noticeably expanding, driven by discovery of a variety of new molecules involved in the functioning of iron metabolic pathways. Iron homeostasis in normal cells is accurately balanced and tightly regulated by a coordinated functioning of several systems that are responsible for the uptake, intracellular storage, and removal of iron from cells [1]; in cancer cells, however, this balance is frequently and consistently compromised [2,3]. Several recent reports have suggested that an association may exist between altered intracellular iron homeostasis, perturbations in the functioning of proteins involved in the iron-regulatory pathways, and breast cancer [4-8]. Thus far, the conclusions have been conflicting and unconvincing. The main question as to whether or not detection of these abnormalities in iron-related metabolic pathways can be used as therapeutic targets for clinical management of breast cancer remains unresolved.

\section{The article}

In a recent issue of Science Translational Medicine, researchers at Wake Forest University School of

Correspondence: igor.pogribny@fda.hhs.gov

Division of Biochemical Toxicology, National Center for Toxicological Research, Jefferson, AR 72079, USA
Medicine demonstrated a critical role of ferroportin in human breast cancer [9]. The authors reported a marked decrease in the levels of ferroportin, the only known mechanism responsible for the export of intracellular non-heme-associated iron to date, in human breast cancer cell lines and breast cancer tissue. Down-regulation of ferroportin was accompanied by up-regulation of hepcidin, which has emerged recently as the central regulator of iron homeostasis in general, and as a key negative regulator of ferroportin in particular [10]. Hepcidin is a 25 amino acid liver-synthesized hormone that inhibits cellular efflux of iron by binding to ferroportin and causing its subsequent degradation $[10,11]$.

More importantly, the investigators showed that a greater magnitude of ferroportin reduction was observed in more aggressive breast cancer subtypes, and that low ferroportin levels were significantly correlated with other well-established prognostic markers for poor breast cancer outcome, such as absence of estrogen receptors, high histological grade, and spread of disease to the lymph nodes. Based on these findings, Pinnix and colleagues [9] determined that ferroportin expression predicts the clinical outcome of breast cancer. The obtained results demonstrate clearly that low ferroportin gene expression was associated with poor breast cancer prognosis, which was evidenced by a significant reduction in metastasis-free and disease-specific survival. In contrast, high ferroportin expression was associated with a better outcome, especially in a breast cancer patient 
population with a combination of high ferroportin and low hepcidin expression. In this cohort of breast cancer patients, 5-year and 10-year distant metastasis-free survival was $95 \%$ and $91 \%$, respectively.

\section{The viewpoint}

Breast cancer is the most prevalent malignancy in women. The success of breast cancer treatment relies on the ability to detect the disease and correct molecular abnormalities at an early stage of disease development. In this respect, the results of the study conducted by Pinnix and coworkers are quite remarkable. First, they demonstrated convincingly the incredible diagnostic and prognostic value of ferroportin and hepcidin gene expression in breast cancer. Additionally, they suggested that determination of these two molecular markers may be used as guidance toward individualized therapy for breast cancer patients, which is a foremost goal of medicine in the 21st century.

Second, results of their study demonstrating that increased concentrations of ferroportin inhibited growth of cancer xenografts in mice open new targeted and mechanism-based therapeutic approaches for breast cancer treatment. This is further supported by evidence that breast cancer patients with a combination of high ferroportin and low hepcidin expression had extremely favorable prognosis for the disease outcome. Therefore, therapeutic manipulations aimed to increase ferroportin levels in breast cancer cells may substantially improve the efficacy of breast treatment. Until now, most research directed toward modulating aberrant iron homeostasis in cancer cells has been focused on the development and usage of specific iron chelators for treatment of cancer [12]. However, targeted suppression of iron importer genes - for example, transferrin receptors [8] - or enforced expression of iron exporter genes - for example, ferroportin [9] - offer a mechanism-based strategy for the treatment of cancer, particularly for breast cancer $[8,9]$. Specifically, results of the study suggest that either direct modulation of ferroportin levels or indirect modulation of it through down-regulation of liver-specific hormone hepcidin may be useful for the treatment of patients with breast cancer. For instance, therapeutic strategies aimed at hepcidin depletion by neutralizing antibodies, hepcidin small interfering RNAs, microRNAs, or inhibitors of the stimulatory pathways for hepcidin expression - for example, CEBPa and/or CEBPb $[13,14]$ - could be potential candidates.

Although further studies are required to define the mechanisms underlying the aberrant functioning of the hepcidin-ferroportin pathway in breast cancer and to determine how modulation of this pathway affects iron homeostasis not only in cancer cells but also in normal cells, the findings of Pinnix and colleagues could facilitate the development of novel markers and therapeutic applications for breast cancer diagnosis and treatment.

\section{Acknowledgements}

The views expressed in this paper do not necessarily represent those of the US Food and Drug Administration.

\section{Competing interests}

The authors declare that they have no competing interests.

Published: 6 October 2010

\section{References}

1. Hentze MW, Muckenthaler MU, Galy B, Camaschella C: Two to tango: regulation of mammalian iron metabolism. Cell 2010, 142:24-38.

2. Richardson DR, Ponka P: The molecular mechanisms of the metabolism and transport of iron in normal and neoplastic cells. Biochim Biophys Acta 1997, 1331:1-40

3. Kwok JC, Richardson DR: The iron metabolism of neoplastic cells: alterations that facilitate proliferation? Crit Rev Oncol Hematol 2002, 42:65-78.

4. Shpyleva SI, Tryndyak VP, Kovalchuk O, Starlard-Davenport A, Chekhun VP, Beland FA, Pogribny IP: Role of ferritin alterations in human breast cancer cells. Breast Cancer Res Treat 2010.

5. Lieghr JG, Jones JS: Role of iron in estrogen-induced cancer. Curr Med Chem 2001, 8:839-849.

6. Kabat GC, Rohan TE: Does excess iron play a role in breast carcinogenesis? An unresolved hypothesis. Cancer Causes Control 2007, 18:1047-1053.

7. Huang X: Does iron have a role in breast cancer? Lancet Oncol 2008, 9:803-807.

8. Jiang XP, Elliot RL, Head JF: Manipulation of iron transported genes results in the suppression of human and mouse mammary adenocarcinomas. Anticancer Res 2010, 30:759-765.

9. Pinnix ZK, Miller LD, Wang W, D'Agostino R Jr, Kute T, Willingham MC, Hatcher H, Tesfay L, Sui G, Di X, Torti SV, Torti FM: Ferroportin and iron regulation in breast cancer progression and prognosis. Sci Transl Med 2010, 2, 43ra56.

10. Fleming MD: The regulation of hepcidin and its effects on systemic and cellular iron metabolism. Hematology Am Soc Hematol Educ Program 2008, 151-158.

11. Franchini M, Montagnana M, Lippi G: Hepcidin and iron metabolism: from laboratory to clinical implications. Clin Chim Acta 2010, 411:1565-1569.

12. Richardson DR, Kalinowski DS, Lau S, Jansson PJ, Lovejoy DB: Cancer cell iron metabolism and the development of potent chelators as antitumour agents. Biochim Biophys Acta 2009, 1790:702-717.

13. Courselaud B, Pigeon C, Inoue Y, Inoue J, Gonzalez FJ, Leroyer P, Gilot D, Boudjema K, Guguen-Guillouzo C, Brissot P, Loréal O, Ilyin G: C/EBPalpha regulates hepatic transcription of hepcidin, an antimicrobial peptide and regulator of iron metabolism. Cross-talk between C/EBP pathway and iron metabolism. J Biol Chem 2002, 277:41163-41170.

14. Sow FB, Alvarez GR, Gross RP, Satoskar AR, Schlesinger LS, Zwilling BS, Lafuse WP: Role of STAT1, NF-kappaB, and C/EBPbeta in the macrophage transcriptional regulation of hepcidin by mycobacterial infection and IFN-gamma. J Leukoc Biol 2009, 86:1247-1258.

doi: $10.1186 /$ bcr2641

Cite this article as: Pogribny: Ferroportin and hepcidin: a new hope in diagnosis, prognosis, and therapy for breast cancer. Breast Cancer Research 2010 12:314. 